\title{
Inkasso - Immer mehr säumige Schuldner?
}

Frau Fankhauser ist seit 1. April 1994 Leiterin der FMH Inkasso Services. Die FMH Inkasso Services ist eine Tochtergesellschaft der FMH Services. Sie sind die Spezialisten und entlasten die Ärzteschaft bei Inkassosorgen.

Stichwort schleppende Zahlungsmoral Wie schätzen Sie die heutige Situation bezüglich Zahlungsmoral gegenüber Ärzten und Ärztinnen ein?

Die anhaltend angespannte Wirtschaftslage dürfte auch bei der Ärzteschaft zu einem erhöhten Debitorenausstand führen. Menschen mit bereits angespannten finanziellen Verhältnissen werden früher oder später auch bei ihrem Arzt säumig. Die Ursache für den Zahlungsverzug oder die Zahlungsverweigerung spielt dabei eine grosse Rolle. Jedenfalls stellen wir in unserer täglichen Arbeit fest, dass sehr viel mehr Zeit und Verhandlungsgeschick eingesetzt werden muss, um Zahlungen erwirken zu können. Fruchtlose Pfändungen infolge von ungenügendem Einkommen und Vermögen sind sehr häufig.

Mit wie vielen Schuldnerdossiers werden Sie im Durchschnitt pro Jahr beauftragt?

Im Jahre 2002 sind uns für ein Honorarvolumen von rund Fr. 9,8 Mio. Aufträge erteilt worden. Das sind ungefähr 19000 Fälle, die von unserem 15köpfigen Team zu bearbeiten sind. Wir sind gesamtschweizerisch tätig, wobei der Grossteil der Aufträge aus der Deutschschweiz stammt.

Welches sind präventive Massnahmen, um ausstehende Honorare zu minimieren? Zunächst empfehlen wir, dass die Leistungen möglichst rasch fakturiert werden. Dann spielt natürlich die Debitorenkontrolle eine ganz wichtige Rolle. Fakturierte Leistungen sollten regelmässig kontrolliert und konsequent gemahnt werden.

\section{Man hört von Patienten/Patientinnen,} die ihre Rechnung nie zahlen, dafür das Geld von ihrer Krankenkasse «einkassieren». Wissen Sie von solchen Fällen und was kann der Arzt oder die Ärztin tun, um dies zu verhindern? Ja, das ist leider sehr häufig der Fall. Gerade Menschen, die schon stark verschuldet sind und laufend betrieben werden und dadurch auf dem es sich dabei um Betrug handle; dies ist leider nicht der Fall. Das Geld wird zwar zweckentfremdet, aber ein Straftatbestand im Sinne des Strafgesetzes ist damit nicht erfüllt.

Was kann der Arzt dann dagegen tun? Der Arzt/die Ärztin, dessen Patient systematisch den Rückerstattungsbetrag zweckentfremdet und für seine eigenen Bedürfnisse verwendet, kann sich um eine Abtretungsvereinbarung mit dem Patienten bemühen. Das Obligationenrecht (Art. 164ff) gestattet es nämlich, dem Gläubiger, eine Forderung abzutreten. Krankenversicherer können sich einer Zession nur dann widersetzen, wenn sie diese in ihren allgemeinen Geschäftsbedingungen entweder gänzlich ausgeschlossen oder von ihrer Zustimmung abhängig gemacht haben. Besteht keine schriftliche Abrede, hat der Patient grundsätzlich das Recht, seinen Rückerstattungsanspruch abzutreten. Die Kasse kann sich dagegen nicht wehren. Sie kann allerdings allfällige ihr gegenüber dem Patienten zustehende Einreden (Verrechnungseinrede) auch gegenüber einem neuen Gläubiger geltend machen und eine solche Forderung z.B. durch Verrechnung mit Prämienausständen zum Untergang bringen.

In welchen Abständen sollte denn eigentlich gemahnt werden?

Gesetzlich gibt es keine Vorschrift, wie und wie oft ein säumiger Schuldner gemahnt werden muss. Für eine spätere Verzugszinsbelastung muss er jedoch zuerst in Verzug gesetzt werden, was in der Regel mit der ersten Mahnung geschieht. Wir empfehlen, die erste Mahnung etwa 10 Tage nach Ablauf der Zahlungsfrist gemäss Rechnungsstellung zu versenden. Jede Mahnung soll eine klare Zahlungsfrist enthalten (z.B. innert 20 Tagen oder innert 30 Tagen). Daraus ergeben sich dann auch die Termine für die weiteren Mahnungen.

Wie viele Mahnstufen gibt es grundsätzlich? Auch dafür gibt es keine gesetzlichen Vorschriften. Usanz ist häufig, dass drei Mahnungen ausgestellt werden. Unsere Erfahrungen zeigen jedoch, dass die dritte Mahnung oft verlorene Mühe ist. Hat sich ein Schuldner nicht auf die erste und zweite Mahnung gemeldet, wird er dies in der Regel auch auf die dritte Mahnung nicht tun. Deshalb ist es völlig ausreichend, zwei Mahnstufen einzusetzen und bereits mit der zweiten Mahnung betreibungsrechtliche Schritte anzu- 
drohen, falls dieser Aufforderung nicht Folge geleistet werden sollte. Die letzte Mahnung muss übrigens nicht zwingend per Einschreiben (Lettre signature) an den Patienten zugestellt werden!

\section{Zu welchem Zeitpunkt wird Ihnen dann der Inkassoauftrag erteilt?}

Dies steht in direktem Zusammenhang mit dem praktizierten Mahnsystem und den Mahnstufen, die eingesetzt werden. Unabhängig davon, ob dem Patienten schon mit der zweiten oder erst mit der dritten Mahnung die Betreibung angedroht wurde - wichtig ist einzig, dass der Arzt/ die Ärztin dann auch «ernst» macht. An «leere» Drohungen gewöhnt sich der Patient dann sehr schnell und er missachtet spätere Mahnungen und Drohungen.

Wie muss ein Arzt oder eine Ärztin den Auftrag erteilen? Wie ist hier das Vorgehen?

Wenn in der Praxis der PC eingesetzt wird, kann man uns einen Ausdruck mit den im Auftragsformular aufgeführten Daten zukommen lassen oder man benutzt unser Auftragsformular. Wenn der Arzt/die Ärztin mit einer externen Stelle abrechnet (z. B. mit der Ärztekasse), kann er diese beauftragen, uns die offenen Forderungen zum Inkasso zu übergeben.

Lohnt es sich, für jeden Betrag eine Betreibung einzuleiten? Und wo sehen Sie die Grenzen?

So pauschal lässt sich diese Frage leider nicht beantworten. Ob ein Betreibungsverfahren zum Erfolg führt oder nicht, ist in erster Linie vom Verhalten und den finanziellen Verhältnissen des
Schuldners abhängig. Leute, die sich über Jahre hinaus in der «Betreibungsspirale» befinden, sind häufig gar nicht in der Lage, die laufenden Verpflichtungen lückenlos zu erfüllen und müssen ihre Gläubiger wohl oder übel über das Betreibungsverfahren befriedigen. Andererseits kommt es darauf an, welches Kosten- und Verlustrisiko der Arzt/die Ärztin bereit ist einzugehen.

Gemäss unseren allgemeinen Geschäftsbedingungen leiten wir die Betreibung grundsätzlich erst dann ein, wenn der Forderungsbetrag mindestens Fr. 200.- beträgt. Zeigen aber unsere Erhebungen, dass das Betreibungsverfahren ohne Ergebnis enden wird, raten wir auch dann von einem Verfahren ab. Hier kann dann nur mit einvernehmlichen Lösungen gearbeitet werden, vorausgesetzt, der Schuldner ist verhandlungsbereit und gewillt, den Verpflichtungen gegenüber seines Arztes nachzukommen.

\section{Wie sehen Sie die Ausrichtung/Zukunft} des Inkassowesens in bezug auf die Ärzteschaft? Ich glaube, dass auch die Ärzteschaft immer mit säumigen Patienten und Patientinnen konfrontiert sein wird. Der Arzt, als Selbständigerwerbender trägt heute mehr denn je ein gewisses «Unternehmerrisiko». Sein Beruf macht es aber natürlich schwierig, vorerst die Solvenz seiner «Kunden» zu prüfen, bevor er die Behandlung beginnt. Ein geschickt eingesetztes Mahnsystem, eine effiziente Debitorenkontrolle und nicht zuletzt natürlich ein professioneller Inkassopartner kann grossen Schaden verhindern und gleichzeitig die notorischen Nichtzahler «aussondern».

\section{FMH SERVICES}

Ihr Partner, wenn Zahlungseingänge sich verzögern!

Säumige Patientinnen und Patienten? Wenn die Zahlungsmoral krankt, ist Erfahrung gefragt. Wir sind die Spezialisten, entlasten Sie von Inkassosorgen und treiben für Sie die Honorarforderungen ein.

Und all dies zu fairen Konditionen, mit grosser Sensibilität und mit der gefragten Kompetenz. Damit Sie sich aufs Wesentliche konzentrieren können. Zum Wohl Ihrer Patientinnen und Patienten.

Votre partenaire pour le règlement de vos factures!

Avez-vous des patients mauvais payeurs? Si cela vous irrite, faites appel à notre expérience. Nous sommes des spécialistes, nous nous occupons de vos soucis de contentieux et encaissons pour vous les honoraires dus!

Et tout ceci à des conditions équitables, avec tact et compétence. Pour que vous puissiez vous concentrer sur l'essentiel. Pour le bien des vos patients et patientes.

\section{FMH INKASSO}

FMH Inkasso Services - Thorackerstrasse 3 - 3074 Muri b. Bern

Tel. 0319508030 - Fax 0319508040 - E-Mail: mail@fmhinkasso.ch 